\title{
Correlation between optical and electrical properties of Mg-doped AIN epilayers
}

\author{
M. L. Nakarmi, N. Nepal, C. Ugolini, T. M. Altahtamouni, J. Y. Lin, and H. X. Jiang ${ }^{\text {a) }}$ \\ Department of Physics, Kansas State University, Manhattan, Kansas 66506-2601
}

(Received 16 May 2006; accepted 29 August 2006; published online 13 October 2006)

\begin{abstract}
Deep UV photoluminescence and Hall-effect measurements were employed to characterize Mg-doped AlN grown by metal organic chemical vapor deposition. A strong correlation between the optical and electrical properties was identified and utilized for material and $p$-type conductivity optimization. An impurity emission peak at $4.7 \mathrm{eV}$, attributed to the transition of electrons bound to triply charged nitrogen vacancies to neutral magnesium impurities, was observed in highly resistive epilayers. Improved conductivity was obtained by suppressing the intensity of the $4.7 \mathrm{eV}$ emission line. $\mathrm{Mg}$-doped AlN epilayers with improved conductivities predominantly emit the acceptor-bound exciton transition at $5.94 \mathrm{eV}$. From the Hall-effect measurements performed at elevated temperatures, the activation energy of $\mathrm{Mg}$ in $\mathrm{AlN}$ was measured to be about $0.5 \mathrm{eV}$, which is consistent with the value obtained from previous optical measurements. Energy levels of nitrogen vacancies and $\mathrm{Mg}$ acceptors in Mg-doped AlN have been constructed. (C) 2006 American Institute of Physics. [DOI: 10.1063/1.2362582]
\end{abstract}

Aluminum nitride (AIN), the largest direct band gap material $(6.1 \mathrm{eV})$ in the InGaAlN family, has attracted much attention recently due to its promising applications in the development of chip-scale optoelectronic devices, such as emitters and detectors in the ultraviolet (UV) spectral range down to $200 \mathrm{~nm}$. For such applications, obtaining device quality materials is essential. We have previously demonstrated that AIN epilayers with an optical quality comparable to $\mathrm{GaN}$ can be grown on sapphire by metal organic chemical vapor deposition (MOCVD). ${ }^{1-3}$ Due to its high thermal conductivity and transparency in the deep ultraviolet (DUV) spectral range $(\lambda>200 \mathrm{~nm})$, AlN epilayers have been widely employed as templates for the development of DUV light emitting diodes with emission wavelengths $\lambda<300 \mathrm{~nm}^{4-9}$

For applications using $\mathrm{AlN}$ or AlGaN with an extremely high Al content as an active material, highly conductive $n$-type and $p$-type AlN materials are desirable. However, it is very difficult to improve the conductivity of AlN due to the large activation energy of dopants and the simultaneous generation of compensating centers and defects during the crystal growth. Recently, progress has been made on achieving $n$-type conductive AlN by Si doping. ${ }^{10-13}$ The thermal activation energy of Si donors in AlN has been determined to be in the range of $86-250 \mathrm{meV}$. For Al-rich AlGaN alloys and AlN, theoretical works have indicated that compensation of electrons by intrinsic defects such as cation vacancies $\left(V_{\mathrm{III}}\right)^{3-}$ or their complexes is responsible for the reduced $n$-type conductivity. ${ }^{14-16}$ However, enhancing the $p$-type conductivity of Al-rich AlGaN alloys is more challenging. We have previously reported the growth and electrical/optical properties of $p$-type $\mathrm{Al}_{x} \mathrm{Ga}_{1-x} \mathrm{~N}$ alloys for $x$ up to $0.27 .{ }^{17}$ More recently, we have also studied the electrical and optical properties and confirmed $p$-type conduction with a resistivity of about $40 \Omega \mathrm{cm}$ in $\mathrm{Mg}$-doped $\mathrm{Al}_{0.7} \mathrm{Ga}_{0.3} \mathrm{~N}$ alloys at $800 \mathrm{~K}$. We previously also reported the optical studies of $\mathrm{Mg}$-doped AlN epilayers and obtained an energy level of $\mathrm{Mg}$ impurities of about $0.5 \mathrm{eV}$ in AlN. ${ }^{18,19}$ However, no electrical measure-

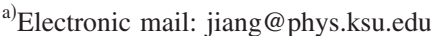

ments could be done in the prior studies, as Mg-doped AlN materials were highly resistive. For the development of high performance DUV emitters and detectors with $\lambda<280 \mathrm{~nm}$, it is imperative to investigate the limitation and methods of p-type doping in Al-rich AlGaN alloys and pure AlN.

We report here on the growth by MOCVD and studies of the correlation between the electrical and optical properties of Mg-doped AlN epilayers. Optical transitions involving the intrinsic defects that act as compensating centers for holes were identified. It was found that the overall material quality and conductivity of Mg-doped AIN are strongly correlated with the emission intensity of the defect related transition. Besides the large activation energy of the $\mathrm{Mg}$ acceptors in AlN, we believe that compensation of holes by "donorlike" intrinsic defects is another cause for the highly resistive nature of Mg-doped AlN.

Mg-doped AlN epilayers of thickness of about $1 \mu \mathrm{m}$ were grown on AlN/sapphire templates by MOCVD. Undoped AlN epilayers of thickness $\sim 0.5 \mu \mathrm{m}$ were first grown on the $c$ plane (0001) of sapphire substrates as templates followed by the growth of Mg-doped AlN epilayers. High quality, undoped AlN epilayer template plays an important role to reduce dislocations in the subsequent doped layer. Trimethyl aluminum and blue ammonia were used as sources for aluminum and nitrogen, respectively. For the magnesium dopant, biscyclopentadienyl-magnesium was transported into the reactor. The growth temperature and pressure for $\mathrm{Mg}$ doped AlN epilayers were about $1200^{\circ} \mathrm{C}$ and 40 torr, respectively. V/III ratio was varied in the range of 400010000 and the optimized layers were grown under a V/III ratio of about 8000 . Mg-doping concentration was determined by secondary ion mass spectroscopy (SIMS). As grown $\mathrm{Mg}$-doped AlN samples were highly resistive and all samples were thermally annealed in $\mathrm{N}_{2}$ environment prior to the photoluminescence (PL) and Hall measurements. DUV PL spectroscopy was employed to characterize and study the optical properties. Electrical properties were characterized by the variable temperature Hall-effect measurements $(10 \mathrm{~K}$ $<T<900 \mathrm{~K})$. The typical $\mathrm{x}$-ray diffraction full width at half 


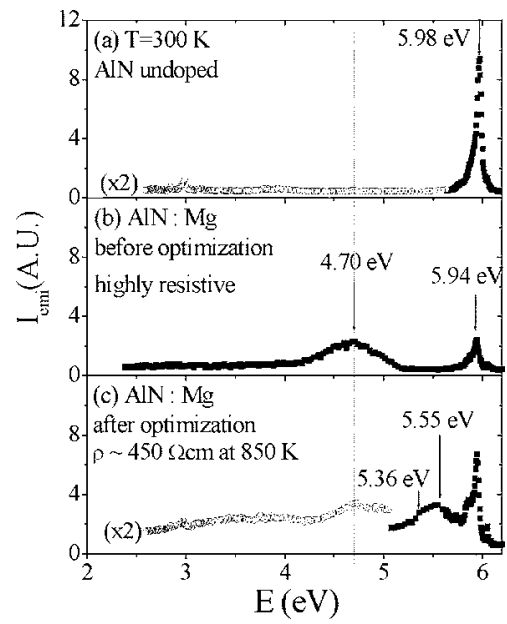

FIG. 1. Room temperature PL spectra of (a) an undoped and highly resistive AlN epilayer, (b) a Mg-doped and high resistive AlN epilayer, and (c) a $\mathrm{Mg}$-doped AlN epilayer with improved conductivity.

maximum of the rocking curves of the (0002) peaks of undoped AlN epilayer templates was between 50 and 100 arc sec.

Figure 1 compares the room temperature $(300 \mathrm{~K}) \mathrm{PL}$ spectra of (a) an undoped AlN epilayer, (b) a Mg-doped AlN epilayer with high resistivity, and (c) a Mg-doped AlN epilayer with improved $p$-type conductivity (or reduced resistivity). The arrows indicate the spectral peak positions. Undoped AlN has a strong band-edge emission peak at $5.98 \mathrm{eV}$ due to the recombination of free excitons ${ }^{1-3}$ and exhibits virtually no impurity transitions in the low energy region, ensuring a good optical quality. Atomic force microscopy revealed an atomically smooth surface with a root mean square roughness of about $7 \AA$ within a $2 \times 2 \mu \mathrm{m}^{2}$ scan. These undoped AIN epilayers were employed as templates for the subsequent growth of Mg-doped AlN epilayers. For $\mathrm{Mg}$ doped AlN epilayers, the PL spectra presented in Figs. 1(b) and 1(c) encompass an emission peak at around $4.7 \mathrm{eV}$, in addition to the band-edge emission at $5.94 \mathrm{eV}$. The bandedge emission peak at $5.94 \mathrm{eV}$ is due to the recombination of excitons bound to neutral $\mathrm{Mg}$ acceptors (or acceptor-bound excitons $\left.I_{1}\right) .{ }^{19}$ The energy separation between the $I_{1}$ and free exciton transitions is $40 \mathrm{meV}$, corresponding to the binding energy $E_{\mathrm{bx}}$ of the acceptor-bound exciton $\left(I_{1}\right)$.

A clear correlation between the electrical and optical properties of $\mathrm{Mg}$-doped AlN epilayers has been observed. Samples exhibiting strong emissions at $4.7 \mathrm{eV}$, such as that shown in Fig. 1(b), are generally highly insulating. By varying the growth conditions, especially the V/III ratio, the intensity of the impurity emission peak at $4.7 \mathrm{eV}$ was significantly suppressed, the $I_{1}$ transition at $5.94 \mathrm{eV}$ was enhanced, and the conductivity was improved. It is also interesting to note that the $I_{1}$ transition in Mg-doped AlN epilayers survives well up to room temperature due to its large binding energy of $40 \mathrm{meV}$. In general, bound exciton transitions are not observable in $\mathrm{Mg}$-doped $\mathrm{GaN}$ at room temperature. For the Mg-doped AIN epilayer with improved conductivity [Fig. 1(c)], additional emission peaks at 5.36 and $5.55 \mathrm{eV}$, which were absent in highly resistive $\mathrm{Mg}$-doped samples, are evident.

The Mg concentration profile in the Mg-doped AlN epilayer has been probed by SIMS and is shown in Fig. 2, which provides evidence of $\mathrm{Mg}$ impurity diffusion toward Downloaded 12 Jul 2010 to 129.118.86.45. Redistribution subject

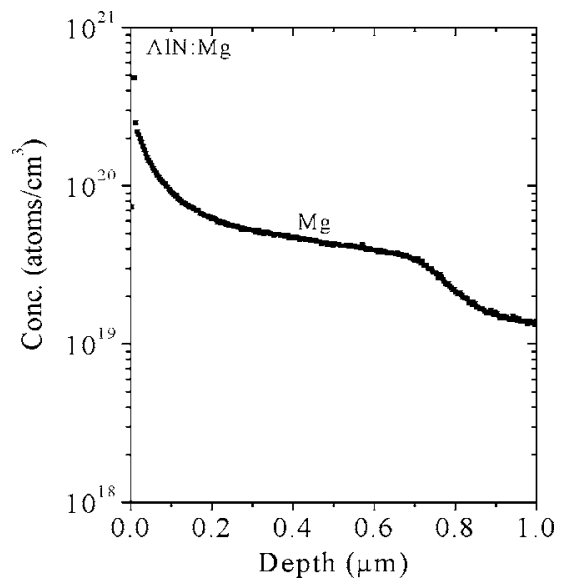

FIG. 2. Profile of magnesium impurities in a Mg-doped AlN epilayer measured by secondary ion mass spectroscopy (SIMS). Mg concentration is on the order of $10^{20} \mathrm{~cm}^{-3}$.

the surface and indicates that the $\mathrm{Mg}$ concentration in the sample is on the order of $10^{20} \mathrm{~cm}^{-3}$. Hall-effect measurements were carried out at elevated temperatures. Figure 3 shows the resistivity for one of our Mg-doped AlN epilayers, in which the intensity of the $4.7 \mathrm{eV}$ emission line was minimized, in the temperature range between 600 and $850 \mathrm{~K}$. At $850 \mathrm{~K}$, the measured $p$-type resistivity is about $450 \Omega \mathrm{cm}$ with a free hole concentration of about $2.5 \times 10^{15} \mathrm{~cm}^{-3}$ and mobility of about $5.5( \pm 3.4) \mathrm{cm}^{2} / \mathrm{V} \mathrm{s}$. At room temperature, the resistivity is estimated to be about $10^{7} \Omega \mathrm{cm}$.

In order to verify that the conduction at high temperatures is due to hole activation from $\mathrm{Mg}$ acceptors, we have obtained the thermal activation energy of the resistivity by fitting the measured temperature dependent resistivity by the relation $\rho(T)=\rho_{0} e^{E_{A} / K T}$, where $\rho(T)$ is the resistivity at temperature $T$, as illustrated in the inset of Fig. 3. The fitted value of $E_{A}$ is about $0.40 \mathrm{eV}$. Assuming a temperature dependent mobility of $\mu \propto T^{-3 / 2}$ at high temperatures, the fitted $E_{A}$ value becomes $0.5 \mathrm{eV}$. This value agrees perfectly with the $\mathrm{Mg}$ acceptor energy level obtained from previous optical measurements. ${ }^{18}$ Thus, the measurable conductivity at high temperatures is due to the conduction of holes.

The intensity of the $4.7 \mathrm{eV}$ emission line was carefully monitored while the growth conditions were varied. A general trend is that a strong emission peak at $4.7 \mathrm{eV}$ translates

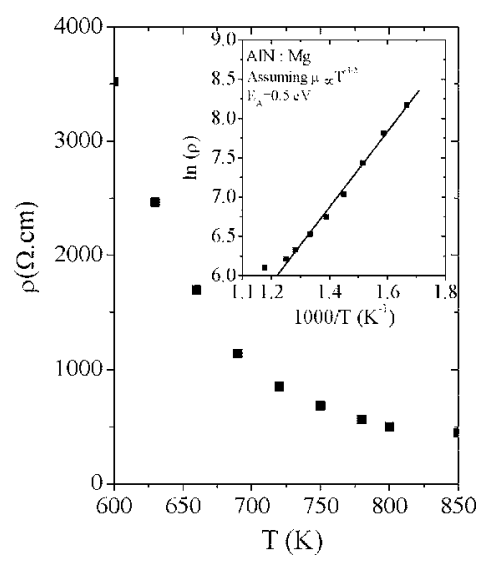

FIG. 3. Temperature variation of the resistivity $(\rho)$ of Mg-doped AlN in the temperature range of $600-850 \mathrm{~K}$. The inset is the Arrhenius plot of $\rho$. The fitted value of the activation energy $E_{A}$ is $0.50 \mathrm{eV}$ assuming a temperature dependent hole mobility $(\mu), \mu \propto T^{-3 / 2}$. 


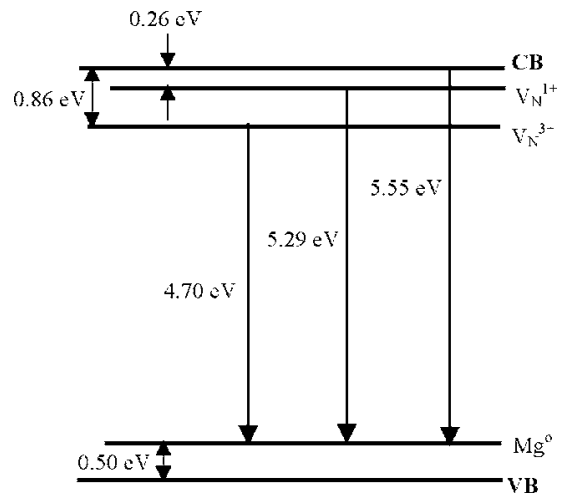

FIG. 4. Energy level diagram of nitrogen vacancies and $\mathrm{Mg}$ acceptors in Mg-doped AlN.

to a highly resistive material even after thermal annealing. The $p$-type conductivity enhances with decreasing the emission intensity of the $4.7 \mathrm{eV}$ line. This correlation clearly indicates that the $4.7 \mathrm{eV}$ emission line is related to hole compensating centers.

Theoretical calculations have indicated that nitrogen vacancies $\left(V_{\mathrm{N}}\right)$ have small formation energy in Mg-doped AlN, which further decreases when the Fermi energy level moves toward the valence band. ${ }^{20}$ The formation energy of nitrogen vacancies with three positive charges $\left(V_{\mathrm{N}}^{3+}\right)$ is smaller than that with one positive charge $\left(V_{\mathrm{N}}^{1+}\right)$. Thus the generation of $V_{\mathrm{N}}^{3+}$ is more favorable during the growth of $\mathrm{Mg}$-doped AlN. $V_{\mathrm{N}}^{3+}$ deep donors can compensate free holes in acceptordoped materials. Once the density of $V_{\mathrm{N}}^{3+}$ deep donors is minimized by varying the growth conditions such as V/III ratio, the emission intensity of the $4.7 \mathrm{eV}$ line is reduced and concomitantly the conductivity is enhanced. In samples with improved conductivity, more neutral $\mathrm{Mg}$ impurities are also available. This is also evident from the increased emission intensity of the free electron to neutral $\mathrm{Mg}\left(\mathrm{Mg}^{0}\right)$ transition at $5.55 \mathrm{eV}$ and the $I_{1}$ transition at $5.94 \mathrm{eV}$. We assign the emission peak at $5.55 \mathrm{eV}$ to the free electrons to $\mathrm{Mg}^{0}$ transition because the spectral peak position is consistent with the measured $\mathrm{Mg}$ energy level and band gap of AlN $(6.06 \mathrm{eV}-0.50 \mathrm{eV}=5.56 \mathrm{eV})$. Due to its strong correlation with the conductivity, we attribute the emission peak at $4.7 \mathrm{eV}$ to the donor and acceptor pair recombination involving electrons bound to $V_{\mathrm{N}}^{3+}$ deep donors and $\mathrm{Mg}^{0}$ in $\mathrm{Mg}$ doped AlN. The enhanced conductivity is due to the suppressed compensating native defects $\left(V_{\mathrm{N}}^{3+}\right)$ in the optimized samples. Thus, we believe that monitoring the $V_{\mathrm{N}}^{3+}$ related PL emission provides an effective way for AlN material and $p$-type conductivity optimization.

Based on our experimental results, we have constructed an energy level diagram involving $\mathrm{Mg}$ acceptors and hole compensating centers in $\mathrm{Mg}$-doped AlN in Fig. 4. We have taken the band gap of AlN to be $6.06 \mathrm{eV}$ at room temperature and free exciton binding energy to be $80 \mathrm{meV}{ }^{2}$ The magnesium level is $0.50 \mathrm{eV}$ above the valence band. Coulomb interaction term between the ionized donors and acceptors has been neglected in the calculations. From the emission line at $4.7 \mathrm{eV}$, the energy level of $V_{\mathrm{N}}^{3+}$ can be calculated as $E_{D}$ $\approx E_{g}-4.7 \mathrm{eV}-0.50 \mathrm{eV} \approx 0.86 \mathrm{eV}$. Theoretically calculated energy level of $V_{\mathrm{N}}^{3+}$ is about $0.9 \mathrm{eV},{ }^{21}$ which is close to our measured value. We have previously observed a nitrogen vacancy $\left(V_{\mathrm{N}}\right)$ related transition in ion-implanted AlN epilayers and determined a binding energy of about $0.26 \mathrm{eV} .^{22}$ Since this value is smaller than the binding energy of $V_{\mathrm{N}}^{3+}$ observed here, we believe that the nitrogen vacancies in ion-implanted AlN are singly charged state $\left(V_{\mathrm{N}}^{1+}\right)$. By including this level in the energy diagram, a possible transition involving $V_{\mathrm{N}}^{1+}$ and $\mathrm{Mg}^{0}$ would give rise to an emission peak at about $5.29 \mathrm{eV}$. The emission line observed at about $5.36 \mathrm{eV}$ in Fig. 1(c) is close to the spectral peak position of this proposed transition by noticing that the Coulomb interaction term between the donor and acceptor has been neglected.

In summary, we have grown Mg-doped AlN epilayers by MOCVD. A strong correlation between the optical and electrical properties was observed. For highly resistive samples, a strong emission peak at $4.7 \mathrm{eV}$ associated with the transition of electrons bound to $V_{\mathrm{N}}^{3+}$ to neutral $\mathrm{Mg}$ was observed. Enhanced conductivities were obtained in samples that exhibit a suppressed PL emission peak at $4.7 \mathrm{eV}$ and enhanced acceptor-bound exciton emission peak at $5.94 \mathrm{eV}$. The enhanced $p$-type conductivity is attributed to the reduced hole compensation by the donorlike intrinsic defects mainly related to $V_{\mathrm{N}}^{3+}$. From the temperature dependent Hall-effect measurement, a value of $0.5 \mathrm{eV}$ for the $\mathrm{Mg}$ acceptor activation energy in AlN has been obtained, which agrees with the value obtained by previous optical measurements.

This work is supported by a grant from DOE (DE-FG0396ER45604).

${ }^{1}$ J. Li, K. B. Nam, M. L. Nakarmi, J. Y. Lin, and H. X. Jiang, Appl. Phys. Lett. 81, 3365 (2002).

${ }^{2}$ K. B. Nam, J. Li, M. L. Nakarmi, J. Y. Lin, and H. X. Jiang, Appl. Phys. Lett. 82, 1694 (2003).

${ }^{3}$ J. Li, K. B. Nam, M. L. Nakarmi, J. Li, J. Y. Lin, H. X. Jiang, P. Carrier, and S. H. Wei, Appl. Phys. Lett. 83, 5163 (2003).

${ }^{4}$ V. Adivarahan, S. Wu, J. P. Zhang, A. Chitinis, M. Shatalov, V. Mandavilli, R. Gaska, and M. Asif Khan, Appl. Phys. Lett. 81, 4910 (2002).

${ }^{5}$ A. Yasan, R. McClintock, K. Mayes, D. Shiell, L. Gautero, S. R. Darvish, P. Kung, and M. Razeghi, Appl. Phys. Lett. 83, 4701 (2003).

${ }^{6}$ A. Hanlon, P. M. Pattison, J. F. Kaeding, R. Sharma, P. Fini, and S. Nakamura, Jpn. J. Appl. Phys., Part 2 42, L628 (2003).

${ }^{7}$ A. J. Fischer, A. A. Allerman, M. H. Crawford, K. H. A. Bogart, S. R. Lee, R. J. Kaplar, W. W. Chow, S. R. Kurtz, K. W. Fullmer, and J. J. Figiel, Appl. Phys. Lett. 84, 3394 (2004).

${ }^{8}$ K. H. Kim, Z. Y. Fan, M. Khizar, M. L. Nakarmi, J. Y. Lin, and H. X. Jiang, Appl. Phys. Lett. 85, 4777 (2004).

${ }^{9}$ M. L. Nakarmi, K. H. Kim, M. Khizar, Z. Y. Fan, J. Y. Lin, and H. X. Jiang, Appl. Phys. Lett. 86, 092108 (2005).

${ }^{10}$ Y. Taniyasu, M. Kasu, and N. Kobayashi, Appl. Phys. Lett. 81, 1255 (2002).

${ }^{11}$ M. L. Nakarmi, K. H. Kim, K. Zhu, J. Y. Lin, and H. X. Jiang, Appl. Phys. Lett. 85, 3769 (2004).

${ }^{12}$ Y. Taniyasu, M. Kasu, and T. Makimoto, Appl. Phys. Lett. 85, 4672 (2004).

${ }^{13}$ T. Ive, O. Brandt, H. Kostial, K. J. Friedland, L. Daweritz, and K. H. Ploog, Appl. Phys. Lett. 86, 024106 (2005).

${ }^{14}$ C. G. Van de Walle and J. Neugebauer, J. Appl. Phys. 95, 3851 (2004).

${ }^{15}$ T. Mattila and R. M. Nieminen, Phys. Rev. B 55, 9571 (1997).

${ }^{16}$ C. Stampfl and C. G. Van de Walle, Appl. Phys. Lett. 72, 459 (1998).

${ }^{17} \mathrm{~J}$. Li, T. N. Oder, M. L. Nakarmi, J. Y. Lin, and H. X. Jiang, Appl. Phys. Lett. 80, 1210 (2002).

${ }^{18}$ K. B. Nam, M. L. Nakarmi, J. Li, J. Y. Lin, and H. X. Jiang, Appl. Phys. Lett. 83, 878 (2003).

${ }^{19}$ N. Nepal, M. L. Nakarmi, K. B. Nam, J. Y. Lin, and H. X. Jiang, Appl. Phys. Lett. 85, 2271 (2004).

${ }^{20}$ C. Stampfl and C. G. Van de Walle, Phys. Rev. B 65, 155212 (2002).

${ }^{21}$ T. L. Tansley and R. J. Egan, Phys. Rev. B 45, 10942 (1992).

${ }^{22}$ N. Nepal, K. B. Nam, M. L. Nakarmi, J. Y. Lin, H. X. Jiang, J. M. Zavada, and R. G. Wilson, Appl. Phys. Lett. 84, 1090 (2004). 